\title{
Manejo de la infección por Helicobacter pylori: apreciación crítica de la literatura
}

\section{Treatment of the Helicobacter pylori Infection: A Critical Appraisal} of the Literature

\author{
Andrés LASERnA ${ }^{\mathrm{a}}$ \\ Pontificia Universidad Javeriana, Colombia \\ Julián E. Barahona-Correa ${ }^{b}$ \\ Pontificia Universidad Javeriana, Colombia \\ Luz Helena Alba ${ }^{c}$ \\ Universidad El Bosque, Colombia
}

a Médico general, Facultad de Medicina, Pontificia Universidad Javeriana, Bogotá, Colombia.

b Médico general, Facultad de Medicina, Pontificia Universidad Javeriana, Bogotá, Colombia.

c Médica general, Facultad de Medicina, Universidad El Bosque, Bogotá, Colombia. Especialista en Medicina Familiar. Epidemióloga Clínica. Profesora asociada al Departamento de Medicina Preventiva y Social, Pontificia Universidad Javeriana, Bogotá, Colombia.

Cómo citar: Laserna A, Barahona-Correa JE, Alba LH. Manejo de la infección por Helicobacter pylori: apreciación crítica de la literatura. Univ. Med. 2018;59(3). doi: https://doi.org/10.11144/Javeriana. umed59-3.pylo

\section{RESUMEN}

Introducción: la infección por Helicobacter pylori es un problema de salud pública, dada su relación con cáncer gástrico. El incremento de la resistencia bacteriana limita la erradicación efectiva, a pesar del empleo de diferentes esquemas de tratamiento. Métodos: revisión de la literatura en la base de datos Pubmed/Medline entre el 1 de enero de 2015 y el 31 de diciembre de 2016 sobre el manejo del Helicobacter pylori. Resultados: se incluyeron 26 artículos. La terapia secuencial sobresale como opción de tratamiento de primera línea para escenarios como Colombia. La implementación de coadyuvantes puede influir en las tasas de erradicación. Los estudios de epidemiología local y costoefectividad son escasos. Conclusiones: el uso y conocimiento adecuado de los esquemas de manejo puede disminuir los costos para el sistema, la resistencia antimicrobiana y favorecer la erradicación de patógenos. Se requieren estudios para generar recomendaciones locales.

Palabras clave

Helicobacter pylori ; tratamiento farmacológico; terapia combinada; guías de práctica clínica como asunto; antibacterianos.

\begin{abstract}
Introduction: Helicobacter pylori (H. pylori) infection is a public health problem due to its relationship with gastric cancer. The escalation of antibiotic resistance hampers an effective eradication, despite the availability of treatment options. Methods: A review of the literature was performed in the database PubMed between 01/01/2015 and 31/31/2016. Results: Twenty six articles were included. Sequential therapy stands out as a first line therapy for scenarios such as Colombia. The implementation of adjuvants may have a positive impact on eradication rates. Local epidemiology and cost-effectiveness studies are scarce. The results were analized by erradication therapies, coadyuvant treatment, guidelines and outcomes non mentioned in the guidelines. Conclusions: The correct use and knowledge of the different treatment options could reduce the costs for the health systems, the antibiotics resistance and could favor pathogen eradication. Further studies are required for establishing local recommendations.
\end{abstract}


Andrés Laserna, Julián E. Barahona-Correa, Luz Helena Alba.

Keywords

Helicobacter pylori; disease management; therapeutics; disease eradication; anti-bacterial agents.

\section{Introducción}

El Helicobacter pylori (H. pylori) es el causante de la infección crónica bacteriana más común en humanos y constituye un problema de salud pública (1). Este microorganismo se asocia con el desarrollo de úlceras pépticas y linfomas (2) y se considera la causa principal del cáncer gástrico, ya que participa en la secuencia: gastritis crónica, metaplasia intestinal, displasia y cáncer (3). Hasta el $90 \%$ de los casos de cáncer gástrico se asocian a $H$. pylori (4) y los sujetos infectados tienen entre tres y seis veces más riesgo de desarrollar este cáncer, comparados con controles no infectados (5).

La Organización Mundial de la Salud considera a este microorganismo un carcinógeno clase 1 (6). Dada su alta prevalencia en países en desarrollo —que puede superar el 60\% en América Latina (7)-, es fundamental crear e implementar intervenciones que disminuyan la incidencia de la infección y sus complicaciones. Por un lado, el conocimiento exacto de su vía de transmisión vía oral-fecal, oral-oral y gastrooral, a través de alimentos y de agua contaminada, permitirá plantear estrategias preventivas $(8,9,10,11,12,13,14)$; por otro, la vacunación se convierte en una medida de protección específica en plena investigación. En China, un estudio clínico fase 3 de la vacuna ha mostrado resultados esperanzadores (15), lo que ofrece posibilidades de intervenciones preventivas a largo plazo. En el entretanto se debe diagnosticar y tratar la infección tempranamente (16). El Consenso Europeo (Maastricht IV) recomendó la erradicación del H. pylori en personas con 1) úlcera péptica, 2) linfoma tipo MALT, 3) gastritis atrófica, 4) resección gástrica poscáncer, 5) antecedentes familiares en primer grado de cáncer gástrico y 6) preferencia de tratar una infección detectada en una visita médica (17).

Actualmente, el tratamiento más usado es la triple terapia de erradicación (TTE), que incluye: inhibidor de bomba de protones (IBP), claritromicina más amoxacilina o nitromidazoles. Sin embargo, la erradicación con esta opción terapéutica es usualmente inferior al 80\% (18) y dado el aumento en muchos países de la tasa de resistencia del $\mathrm{H}$. pylori, se han propuesto nuevos regímenes como alternativas de primera línea para lograr la tasa de erradicación ideal ( $i$. e. mayor al 90\%) $(19,20,21,22)$.

Las alternativas más estudiadas son 1) la terapia cuádruple con bismuto (TCB); los esquemas sin bismuto: 2) la terapia secuencial (SEC) y 3) la terapia concomitante (CON): TTE más metronidazol o tinidazol; 4) los regímenes híbridos (HIB) (23), y 5) los esquemas con fluoroquinolonas (24) (tabla 1).

\section{Tabla 1}

Esquemas terapéuticos

\begin{tabular}{|c|c|c|}
\hline Esquema & Sigla & $\begin{array}{c}\text { Características } \\
\end{array}$ \\
\hline $\begin{array}{l}\text { Terapia Triple de } \\
\text { Erradicación }\end{array}$ & TTE & $\begin{array}{l}\text { Inhibidor de bomba de protones cada } 12 \mathrm{hs}+\text { amoxicilina } 1 \mathrm{~g} / 12 \mathrm{~h} \\
+(\text { claritromicina de } 500 \mathrm{mg} / 12 \mathrm{~h} \text { o metronidazol de } 500 \mathrm{mg} / 12 \mathrm{~h} \text { ), } \\
\text { durante } 7,10 \mathrm{o} 14 \text { días. }\end{array}$ \\
\hline $\begin{array}{l}\text { Terapia } \\
\text { Cuádruple con } \\
\text { Bismuto }\end{array}$ & TCB & $\begin{array}{l}\text { Inhibidor de bomba de protones cada } 12 \text { horas + subcitrato de } \\
\text { bismuto coloidal de } 120 \mathrm{mg} \text { cada } 6 \mathrm{~h}+\text { tetraciclina de } 500 \mathrm{mg} / 6 \mathrm{~h} \\
+ \text { metronidazol de } 500 \mathrm{mg} \text { cada } 8 \mathrm{hs} \text {. }\end{array}$ \\
\hline $\begin{array}{l}\text { Terapia } \\
\text { Secuencial }\end{array}$ & SEC & $\begin{array}{l}\text { Inhibidor de bomba de protones cada } 12 \mathrm{~h}+\text { amoxicilina de } 1 \mathrm{~g} \\
\text { cada } 12 \mathrm{~h} \text {, durante } 5-7 \text { dias, seguido de inhibidor de bomba de } \\
\text { protones cada } 12 \mathrm{~h}+\text { claritromicina de } 500 \mathrm{mg} \text { cada } 12 \mathrm{~h}+ \\
\text { metronidazol/tinidazol de } 500 \mathrm{mg} \text { cada } 12 \mathrm{~h} \text {, durante } 5-7 \text { dias. }\end{array}$ \\
\hline $\begin{array}{l}\text { Terapia } \\
\text { Concomitante }\end{array}$ & CON & $\begin{array}{l}\text { Inhibidor de bomba de protones cada } 12 \mathrm{~h}+\text { amoxicilina de } 1 \mathrm{~g} \\
\text { cada } 12 \mathrm{~h}+\text { claritromicina de } 500 \mathrm{mg} \text { cada } 12 \mathrm{~h}+ \\
\text { metronidazol/tinidazol de } 500 \mathrm{mg} \text { cada } 12 \mathrm{horas} \text {, por } 5,10 \mathrm{o} 14 \\
\text { dias. }\end{array}$ \\
\hline Terapia Hibrida & HIB & $\begin{array}{l}\text { Inhibidor de bomba de protones cada } / 12 \mathrm{~h}+\text { amoxicilina de } 1 \mathrm{~g} \\
\text { cada } / 12 \mathrm{~h} \text { por } 7 \text { días, seguido de inhibidor de bomba de protones } \\
\text { cada/ } 12 \mathrm{~h}+\text { amoxicilina de } 1 \mathrm{~g} \text { cada } 12 \mathrm{~h}+\text { claritromicina de } 500 \\
\mathrm{mg} \text { cada/ } 12 \mathrm{~h}+\text { metronidazol/tinidazol de } 500 \mathrm{mg} \text { cada/ } 12 \mathrm{~h} \text { por } 7 \\
\text { dias. }\end{array}$ \\
\hline $\begin{array}{l}\text { Terapia con } \\
\text { Fluoroquinolonas }\end{array}$ & $\mathrm{TF}$ & $\begin{array}{l}\text { Inhibidor de bomba de protones cada } / 12 \mathrm{~h}+\text { amoxicilina de } 1 \mathrm{~g} \\
\text { cada/ } / 2 \mathrm{~h}+\text { levofloxacina de } 250 \mathrm{mg} \text { cada/ } 12 \mathrm{~h} \text {, durante } 10-14 \\
\text { días. }\end{array}$ \\
\hline
\end{tabular}

Fuente: adaptado de Otero RW et al. Clinical Practice Guideline for the Diagnosis and Management of Adult Patients with Helicobacter pylori infection. Rev Colomb Gastroenterol. 2015;30(Suppl. 1):17-33.

Debido al reciente dinamismo de las recomendaciones en el tratamiento del $\mathrm{H}$. pylori, se revisaron las nuevas opciones de tratamiento en guías de manejo, revisiones sistemáticas y metanálisis publicados entre 2015 y 2016, con el fin de hacer una actualización y análisis crítico de los avances terapéuticos.

\section{Metodología}

Se revisó la literatura sobre el tema en la base de datos Pubmed/Medline 
usando los términos MeSH (Medical Subjects Headings) Helicobacter pylori, Disease management, Treatment, Therapeutics, Eradication of disease, Eradication, Anti-Bacterial Agents. Se utilizó la combinación que arrojara más resultados y fueron filtrados por tipo de artículo, incluyendo guías, metanálisis y revisiones sistemáticas. Se incluyeron los documentos disponibles en texto completo, publicados entre el 1 de enero de 2015 y el 31 de diciembre de 2016, en humanos y disponibles en inglés, francés, español o portugués.

Los artículos se organizaron en el gestor de referencias Mendeley ${ }^{\circledR}$. Se revisaron los títulos y resúmenes y sólo seleccionaron los referentes al manejo del $H$. pylori. Otros tres artículos se obtuvieron con la metodología de bola de nieve y se agregó la guía de manejo colombiana publicada en el 2015 (25), para poder comparar las recomendaciones de los demás estudios con lo propuesto en Colombia. Los resultados de este proceso se organizaron en cuatro categorías: 1) tratamientos de erradicación, 2) terapias coadyuvantes, 3) recomendaciones provenientes de las guías de manejo y 4) desenlaces no mencionados en las guías de manejo.

\section{Resultados}

De la búsqueda resultaron 50 artículos, de los cuales 28 no se relacionaban con el manejo de la infección, tres artículos se identificaron por el método de la bola de nieve y un artículo se incluyó para analizar las recomendaciones actuales en Colombia para un total de 26 manuscritos (23-48) (figura 1 y tabla 2).

\section{Figura 1}

Prisma de búsqueda y selección de artículos

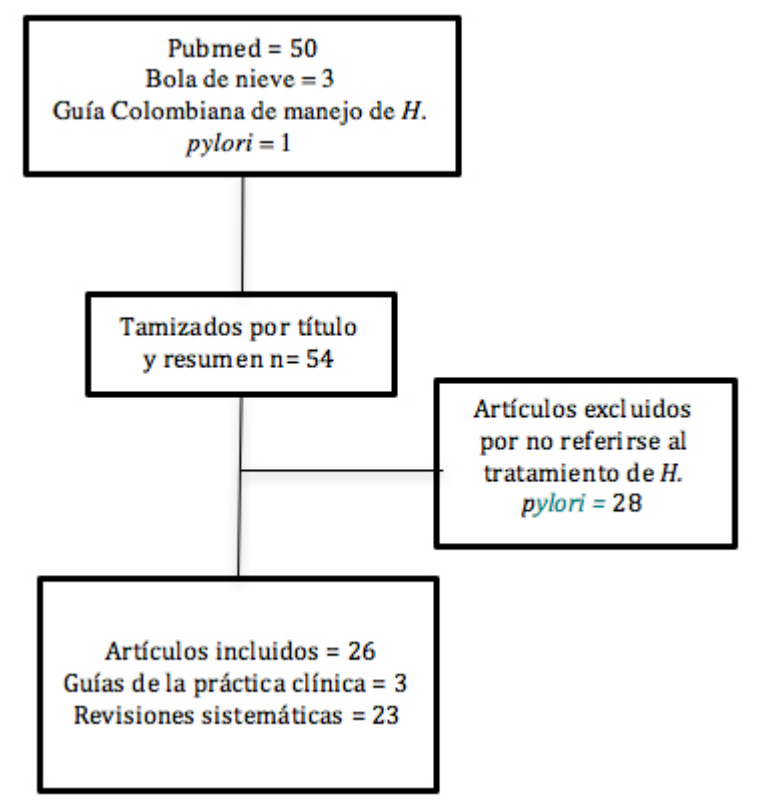

Tabla 2

Artículos incluidos en la revisión

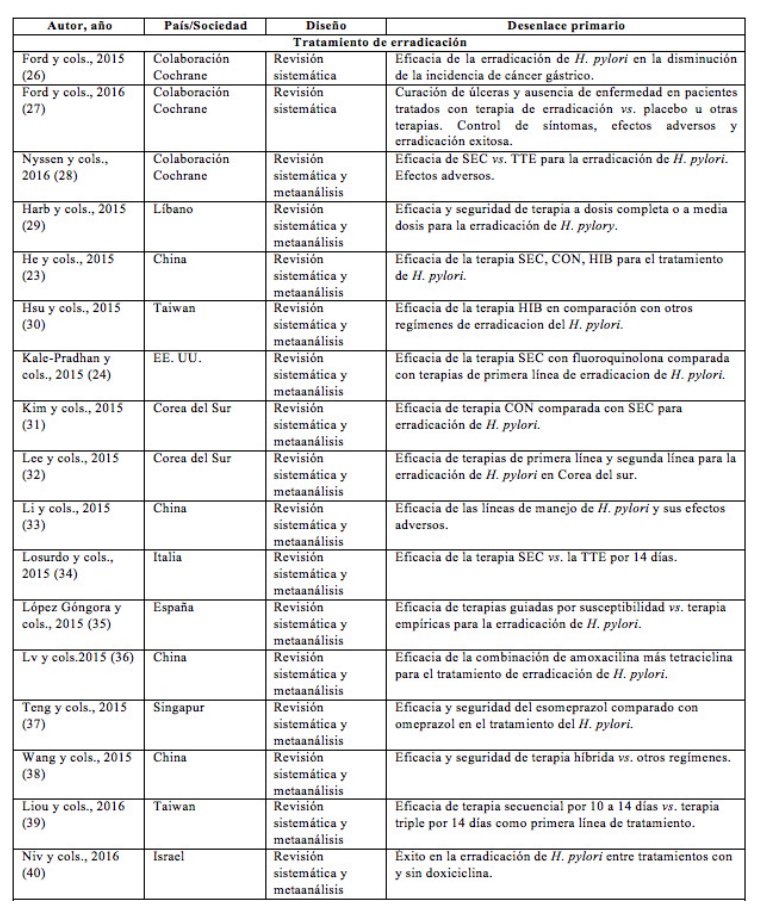


Tabla 2 (cont.)

Artículos incluidos en la revisión

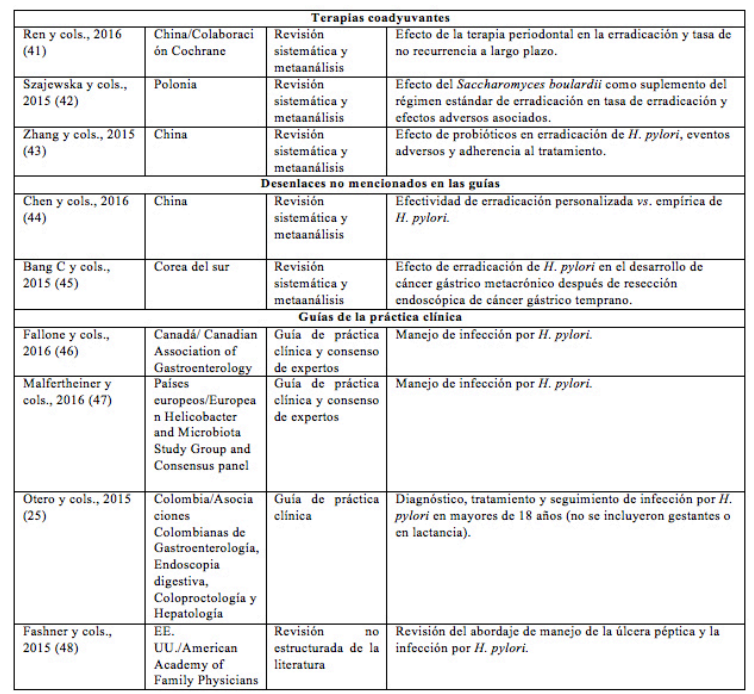

Tratamiento de erradicación

La Colaboración Cochrane realizó tres revisiones sistemáticas. La primera (26) señalaba que el riesgo de cáncer gástrico se reduce en un 34\% con el tratamiento de H. pylori y concluía que la erradicación puede reducir la incidencia futura de cáncer gástrico en personas sanas y asintomáticas infectadas (riesgo relativo [RR]: 0,66; IC95\%: 0,46-0,95); sin embargo, no hay certeza de que lo anterior ocurra en todos los países.

La segunda (27) estableció que los sujetos tratados con terapia de erradicación tenían una disminución significativa del riesgo relativo de recurrencia de úlceras duodenales del $80 \%$ (RR: 0,20; IC95\%: 0,15-0,26) y del 69\% de úlceras gástricas (RR: 0,31; IC95\%: 0,22-0,45), comparado con sujetos no tratados.

La tercera (28) reportó una eficacia significativamente más alta para el tratamiento durante 7 a 10 días con SEC, que aquel realizado con TTE. Sin embargo, en ambos casos, la erradicación se mantuvo menor a lo esperado ( $83 \%$ y $75 \%$, respectivamente). No se encontró superioridad estadística cuando la TTE se administró durante 14 días $(p=0,32)$. La diferencia se explicó dado que la terapia SEC no se vio afectada por la resistencia a claritromicina, lo que favorece que esta terapia se use en lugares con alta resistencia a este medicamento (i.e., > 20\%) (28).

Otros 14 estudios referentes a la terapia de erradicación se realizaron en el último año. Harb y cols. (29) no encontraron diferencia significativa en la eficacia entre la TTE con claritromicina a dosis media $(250 \mathrm{mg})$ y dosis completa $(500 \mathrm{mg})(82 \%$ vs. $83 \%)$ (RR: 0,98; IC95\%: 0,95-1,02). En cuanto a los efectos adversos, se observó que fueron reportados en un $29 \%$ de los pacientes tratados con media dosis y en el $44 \%$ de los que recibieron dosis completa (RR: 0,67; IC95\%: 0,60-0,75).

$\mathrm{He}$ y cols. (23) reportaron que no existía una diferencia estadística entre tres terapias (SEC: 84,3\% [IC95\%: 79,1-89,4]; CON: 86,7\% [IC95\%: 81,0-92,3]; HIB: 86,6\% [IC95\%: 82,3-91,0]). En consecuencia, se analizó el costo de las terapias y se encontró que la SEC mostró ser más económica, pero con la limitante de que el cambio en el régimen generó menos adherencia, dada la complejidad en su administración. Así mismo, la terapia CON fue considerada más sencilla, pudiendo aumentar la adherencia en la práctica clínica. En relación con los efectos adversos, no se encontraron diferencias entre los tres regímenes.

Hsu y cols. (30) encontraron que no hubo diferencia significativa en la erradicación entre TTE, HIB y SEC (RR: 1,01; IC95\%: 0,92-1,11). Por otro lado, reportaron que la terapia HIB fue más efectiva que la terapia SEC en todos los estudios analizados en población no italiana (IC95\%: 1,01-1,18) y menos efectiva en un estudio de población italiana (IC95\%: 0,83-0,98). La resistencia antimicrobiana y la adherencia a los regímenes pudieron explicar las diferencias. La terapia HIB y la CON resultaron ser igual de efectivas (IC95\%: 0,93-1,02).

Kale-Pradhan y cols. (24) reportaron que el uso de fluoroquinolonas podría ser una buena opción de primera línea con tasas de erradicación entre el $73 \%$ y el $97 \%$. Todos los regímenes fueron bien tolerados y no hubo una diferencia estadística entre las terapias. Una gran limitante es que el uso de fluoroquinolonas 
puede aumentar la resistencia de otras bacterias gramnegativas.

Kim y cols. (31) observaron una tendencia a mayor efectividad de la terapia $\mathrm{CON}$ en relación con la terapia SEC, sin significancia estadística (OR: 1,11; IC95\%: 0,79-1,56). Adicionalmente, no hubo disminución en la adherencia con la terapia SEC. La terapia CON, al contar con múltiples medicamentos, supone mayor costo.

Lee y cols. (32) encontraron que la TTE no fue efectiva durante 7 días, comparada con SEC (OR: 0,49; IC95\%: 0,35-0,69), y al prolongarla hasta 14 días no tuvo diferencias significativas (OR: 0,78; IC95\%: 0,31-2,00). Adicionalmente, establecieron que no había información suficiente para tomar decisiones respecto a la terapia $\mathrm{CON}$ y que las terapias con fluoroquinolonas no fueron efectivas.

Li y cols. (33) compararon 14 terapias y reportaron que la menos efectiva fue la TTE durante 7 días. En la medida en que los tratamientos se prolongaron en el tiempo, tuvieron una mayor efectividad; este fue el caso de la terapia CON, que mostró una mejor efectividad, sin ser estadísticamente significativa. De forma interesante, en el estudio se evidenció que la adición de probióticos a la TTE incrementó la efectividad, sobre todo cuando se dieron a lo largo de 7 días. Sin embargo, los estudios evaluados presentaban tamaños de muestra pequeños.

Losurdo y cols. (34) compararon la terapia SEC y 14 días de TTE en lugares con alta y baja resistencia a claritromicina y metronidazol, sin encontrar una diferencia significativa en ninguno de los grupos. Consideraron que, a pesar de la ausencia de diferencias, la terapia SEC fue menos costosa que la TTE, lo que podría representar beneficios.

López-Góngora y cols. (35) observaron que la terapia guiada por sensibilidad fue superior al tratamiento con TTE empírica como primera línea (RR: 1,16; IC95\%: 1,10-1,23), en personas con endoscopia de vías digestivas altas y biopsia. La información para segunda línea de tratamiento fue inconclusa.

Lv y cols. (36) encontraron que las terapias con amoxacilina y tetraciclina no fueron inferiores a los demás regímenes, por lo que podrían usarse como terapias de primera, segunda o tercera línea.

Teng y cols. (37) encontraron una diferencia significativa entre esomeprazol de $40 \mathrm{mg}$ y omeprazol de $20 \mathrm{mg}$ asociados a TTE; sin embargo, los estudios presentaban alta heterogeneidad. Cuando se comparó esomeprazol de $20 \mathrm{mg}$ con omeprazol de $20 \mathrm{mg}$ no hubo diferencia.

Wang y cols. (38) analizaron la seguridad y eficacia de la terapia HIB comparándola con la terapia SEC y CON, sin encontrar diferencias estadísticas. Estos tratamientos podrían considerarse de primera línea.

Liou y cols. (39) encontraron que la terapia SEC entre 10 y 14 días no fue significativamente superior a los 14 días de TTE (RR: 1,04; IC95\%: 0,99-1,08). Sin embargo, reportaron importante heterogeneidad: el análisis de subgrupos mostró que la terapia SEC durante 14 días fue más efectiva que la TTE a lo largo de 14 días (RR: 1,09; IC95\%: 1,04-1,16) y la terapia SEC durante 10 días no fue estadísticamente superior a la TTE por 14 días (RR: 1,03; IC95\%: 0,98-1,09). No se encontraron diferencias significativas en efectos adversos.

Niv (40) no halló diferencias significativas entre los tratamientos con doxiciclina y aquellos con tetraciclina. Sin embargo, cuando los regímenes con doxiciclina fueron comparados con tratamientos sin tetraciclina, se encontró una diferencia favorable de los tratamientos con doxiciclina (OR 1,59; IC95\%: 1,21-2,09).

\section{Terapias coadyuvantes}

En una revisión sistemática de la Colaboración Cochrane (41), se encontró que la terapia periodontal generó un beneficio en el tratamiento del $H$. pylori gástrico. La tasa de erradicación aumentó en los pacientes que recibieron terapia de erradicación más terapia periodontal, comparado con terapia de erradicación única (OR: 2,15; IC95\%: 1,47-3,14). Esto se observó particularmente en pacientes con colonización oral y gástrica por 
H. pylori. Además, los pacientes tratados con terapia periodontal más terapia de erradicación presentaron menos recurrencias (OR: 3,60; IC95\%: 2,11-6,15).

De acuerdo con Szajewska y cols. (42), los pacientes que recibieron suplementos con S. boulardii (probiótico) tuvieron una tasa de erradicación estadísticamente mayor que los controles tratados sin suplementación (80\%; IC95\%: 77-82 vs. 71\%; IC95\%: 68-74) (RR: 1,11; IC95\%: 1,06-1,17). También observaron una disminución significativa en el riesgo de efectos adversos (RR: 0,44; IC95\%: 0,31-0,64), especialmente diarrea (RR: 0,51; IC95\%: 0,420,62) y náusea (RR: 0,6; IC95\%: 0,440,83). Otros efectos adversos como dolor epigástrico, alteraciones gustativas, boca seca, distensión abdominal, frecuencia de vómitos, estreñimiento, otras reacciones inespecíficas o presencia de toxina de $\mathrm{C}$. difficile, no mostraron un cambio significativo (42).

Zhang y cols. (43) reportaron una diferencia estadística en cuanto a las tasas de erradicación cuando se suplementó con probióticos (RR: 1,11; IC95\%: 1,08-1,15). También hubo una reducción significativa en los efectos adversos (RR: 0,59; IC95\%: 0,48-0,71). No se encontraron diferencias en la adherencia al tratamiento (RR: 0,98; IC95\%: 0,68-1,39).

Recomendaciones provenientes de guías de manejo

En el 2015, la Asociación Americana de Médicos Familiares presentó una revisión no estructurada de la literatura sobre el diagnóstico y el tratamiento de las úlceras pépticas y el $\mathrm{H}$. pylori. Recomendaron la terapia CON como primera línea de manejo, argumentando que esta presenta la mayor tasa de erradicación, aunque se reporta que otros regímenes también podrían utilizarse (48).

La Asociación Canadiense de Gastroenterología y el Grupo Canadiense de Estudio del H. pylori realizaron una actualización del manejo en el 2016 (Consenso de Toronto), con 14 recomendaciones. Resaltaron considerar a la terapia SEC como un régimen con eficacia inferior a la terapia CON (46) (tabla 3). Por su parte, el reporte de Maastricht V/Consenso de Florencia V del 2016 generó 20 sugerencias con respecto al manejo (47) (tabla 4). La guía colombiana, por su parte (25), propuso 18 recomendaciones (tabla 5).

\section{Tabla 3}

Recomendaciones del Consenso de Toronto (46)

\begin{tabular}{|c|c|}
\hline \multicolumn{2}{|c|}{ Todos los pacientes } \\
\hline $\begin{array}{l}\text { 1. Se recomienda el tratamiento de pacientes con } \\
\text { H. pylori por un tiempo de } 14 \text { dias. }\end{array}$ & $\begin{array}{l}\text { GRADE: Recomendación fuerte. } \\
\text { Evidencia moderada para TTE y muy } \\
\text { baja para PBMT, PAMC, PAL. }\end{array}$ \\
\hline $\begin{array}{l}\text { 2. Se recomienda que la primera línea de manejo } \\
\text { se escoja según los patrones regionales de } \\
\text { resistencia y las tasas de erradicación. }\end{array}$ & $\begin{array}{l}\text { GRADE: Recomendación fuerte, } \\
\text { calidad de evidencia baja. }\end{array}$ \\
\hline $\begin{array}{l}\text { 3. Se recomienda el uso de la TCB por } 14 \text { dias } \\
\text { como una de las alternativas para la primera línea } \\
\text { de tratamiento. }\end{array}$ & $\begin{array}{l}\text { GRADE: Recomendación fuerte, } \\
\text { calidad de evidencia muy baja para } \\
\text { tiempo y moderada para eficacia. }\end{array}$ \\
\hline $\begin{array}{l}\text { 4. Se recomienda el uso de la terapia CON por } 14 \\
\text { dias como primera línea de tratamiento. }\end{array}$ & $\begin{array}{l}\text { GRADE: Recomendación fuerte, } \\
\text { calidad de evidencia muy baja para } \\
\text { tiempo y moderada para eficacia. }\end{array}$ \\
\hline $\begin{array}{l}\text { 5. Se recomienda restringir el uso de TTE (PCA o } \\
\text { PMC) por } 14 \text { días para áreas con baja resistencia a } \\
\text { la Claritromicina }(<15 \%) \text { o tasas de erradicación } \\
>85 \% \text {. }\end{array}$ & $\begin{array}{l}\text { GRADE: Recomendación fuerte, } \\
\text { calidad de evidencia moderada para } \\
\text { uso de TTE por } 14 \text { dias y baja para } \\
\text { restricciones. }\end{array}$ \\
\hline $\begin{array}{l}\text { 6. Recomendación en contra del uso de triple } \\
\text { terapia con levofloxacina (PAL) como primera } \\
\text { línea de tratamiento. }\end{array}$ & $\begin{array}{l}\text { GRADE: Recomendación fuerte, } \\
\text { calidad de evidencia muy baja. }\end{array}$ \\
\hline $\begin{array}{l}\text { 7. Recomendación en contra del uso de terapia } \\
\text { SEC como primera linea de tratamiento. }\end{array}$ & $\begin{array}{l}\text { GRADE: Recomendación fuerte, } \\
\text { calidad de la evidencia moderada. }\end{array}$ \\
\hline $\begin{array}{l}\text { 8. En pacientes con falla a la terapia de } \\
\text { erradicación, se recomienda el uso de terapia TCB } \\
\text { por } 14 \text { días como terapia siguiente. }\end{array}$ & $\begin{array}{l}\text { GRADE: Recomendación fuerte, } \\
\text { calidad de evidencia baja. }\end{array}$ \\
\hline $\begin{array}{l}\text { 9. En pacientes con falla a la terapia de } \\
\text { erradicación se recomienda el uso de terapias con } \\
\text { levofloxacina por } 14 \text { dias como una opción de } \\
\text { tratamiento. }\end{array}$ & $\begin{array}{l}\text { GRADE: Recomendación } \\
\text { condicionada, calidad de evidencia } \\
\text { baja. }\end{array}$ \\
\hline $\begin{array}{l}\text { 10. En pacientes que han fallado al tratamiento con } \\
\text { regimenes con claritromicina, la recomendación es } \\
\text { en contra de usar terapias que contengan } \\
\text { claritromicina. }\end{array}$ & $\begin{array}{l}\text { GRADE: Recomendación } \\
\text { condicionada, calidad de evidencia } \\
\text { baja. }\end{array}$ \\
\hline $\begin{array}{l}\text { 11. En pacientes que han fallado al tratamiento con } \\
\text { regímenes con levofloxacina hay recomendación } \\
\text { contra usar terapias que contengan levofloxacina } \\
\text { como terapia siguiente. }\end{array}$ & $\begin{array}{l}\text { GRADE: Recomendación } \\
\text { condicionada, calidad de evidencia } \\
\text { baja. }\end{array}$ \\
\hline $\begin{array}{l}\text { 12. En pacientes que han fallado a la terapia de } \\
\text { erradicación, no se recomienda el uso de terapia } \\
\text { SEC como terapia siguiente. }\end{array}$ & $\begin{array}{l}\text { GRADE: Recomendación } \\
\text { condicionada, calidad de evidencia } \\
\text { muy baja. }\end{array}$ \\
\hline $\begin{array}{l}\text { 13. Se recomienda que los regímenes con } \\
\text { rifabutina se restrinjan a los casos en donde } 3 \text { de } \\
\text { las recomendaciones previas hayan fallado. }\end{array}$ & $\begin{array}{l}\text { GRADE: Recomendación } \\
\text { condicionada, calidad de evidencia } \\
\text { muy baja. }\end{array}$ \\
\hline $\begin{array}{l}\text { 14. Recomendación en contra del uso rutinario de } \\
\text { probióticos con la terapia de erradicación, con el } \\
\text { propósito de evitar efectos adversos. }\end{array}$ & $\begin{array}{l}\text { GRADE: Recomendación } \\
\text { condicionada, calidad de evidencia } \\
\text { muy baja. }\end{array}$ \\
\hline $\begin{array}{l}\text { 15. Recomendación en contra del uso de } \\
\text { probióticos con la terapia de erradicación, con el } \\
\text { propósito de aumentar la tasa de erradicación }\end{array}$ & $\begin{array}{l}\text { GRADE: Recomendación } \\
\text { condicionada, calidad de evidencia } \\
\text { muy baja. }\end{array}$ \\
\hline
\end{tabular}




\section{Tabla 4}

Recomendaciones de Maastricht V/Consenso de Florencia V (47)

\begin{tabular}{|c|c|}
\hline Recomendación & Calidad de evidencia \\
\hline $\begin{array}{l}\text { 1. La tasa de resistencia a antibióticos del H. pylori ha } \\
\text { incrementado en muchas partes del mundo. }\end{array}$ & $\begin{array}{l}\text { Recomendación fuerte, nivel } \\
\text { de evidencia moderada. }\end{array}$ \\
\hline $\begin{array}{l}\text { 2. El uso de TTE con IBP y claritromicina, sin previo test } \\
\text { de susceptibilidad, debe ser abandonada en los lugares } \\
\text { donde la resistencia a claritromicina sean }>15 \% \text {. }\end{array}$ & $\begin{array}{l}\text { Recomendación débil, nivel de } \\
\text { evidencia bajo. }\end{array}$ \\
\hline $\begin{array}{l}\text { 3. Para cualquier régimen la tasa de erradicación puede } \\
\text { ser predicha si se conoce la tasa de curación para las } \\
\text { cepas susceptibles y resistentes y la prevalencia de } \\
\text { resistencia en la población. Para un individuo la historia } \\
\text { de cualquier uso previo de uno de los antibióticos clave } \\
\text { propuestos, identificará posiblemente resistencia } \\
\text { antibiótica por más de que exista una baja tasa de } \\
\text { resistencia en la población. }\end{array}$ & $\begin{array}{l}\text { Recomendación fuerte, nivel } \\
\text { de evidencia bajo. }\end{array}$ \\
\hline $\begin{array}{l}\text { 4. En áreas con una alta tasa de resistencia a la } \\
\text { claritromicina ( }>15 \%) \text { se recomienda TCB o CON. En } \\
\text { áreas con alta resistencia a la claritromicina y } \\
\text { metronidazol, la terapia TCB es recomendada como } \\
\text { primera línea. }\end{array}$ & $\begin{array}{l}\text { Recomendación fuerte, nivel } \\
\text { de evidencia bajo. }\end{array}$ \\
\hline $\begin{array}{l}\text { 5. La duración del tratamiento con terapias TCB debe } \\
\text { extenderse a } 14 \text { dias, a menos de que las terapias de } 10 \\
\text { días hayan mostrado efectividad local. }\end{array}$ & $\begin{array}{l}\text { Recomendación débil, nivel de } \\
\text { evidencia bajo. }\end{array}$ \\
\hline $\begin{array}{l}\text { 6. La resistencia a la claritromicina disminuye la eficacia } \\
\text { de la TTE y la terapia SEC y la resistencia a } \\
\text { claritomicina y metronidazol disminuye la eficacia de la } \\
\text { terapia SEC, HIB y CON. }\end{array}$ & $\begin{array}{l}\text { Recomendación fuerte, nivel } \\
\text { de evidencia moderado. }\end{array}$ \\
\hline $\begin{array}{l}\text { 7. Actualmente, la terapia CON debe ser la terapia } \\
\text { cuádruple sin Bismuto preferida, porque ha demostrado } \\
\text { sobrepasar la resistencia antibiótica. }\end{array}$ & $\begin{array}{l}\text { Recomendación fuerte, nivel } \\
\text { de evidencia: moderado. }\end{array}$ \\
\hline $\begin{array}{l}\text { 8. El tiempo de tratamiento recomendado de terapia CON } \\
\text { es } 14 \text { dias, a menos de que las terapias de } 10 \text { dias hayan } \\
\text { sido efectivas localmente. }\end{array}$ & $\begin{array}{l}\text { Recomendación débil, nivel de } \\
\text { evidencia bajo. }\end{array}$ \\
\hline $\begin{array}{l}\text { 9. En áreas con baja resistencia a la claritromicina, la } \\
\text { TTE es recomendada como primera línea de tratamiento; } \\
\text { la terapia TCB es una alternativa. }\end{array}$ & $\begin{array}{l}\text { Recomendación fuerte, nivel } \\
\text { de evidencia alto. }\end{array}$ \\
\hline $\begin{array}{l}\text { 10. El uso de altas dosis de IBP dos veces al día } \\
\text { incrementa la eficacia de la TTE. Esomeprazol y } \\
\text { rabeprazol se prefiere en Europa y Norteamérica, donde } \\
\text { la prevalencia de metabolizadores rápidos es alta. }\end{array}$ & $\begin{array}{l}\text { Recomendación débil, nivel de } \\
\text { evidencia bajo. }\end{array}$ \\
\hline $\begin{array}{l}\text { 11. La duración del tratamiento con terapias basadas en } \\
\text { IBP y claritromicina debe extenderse a } 14 \text { días, a menos } \\
\text { de que terapias más cortas hayan mostrado efectividad } \\
\text { local. }\end{array}$ & $\begin{array}{l}\text { Recomendación fuerte, nivel } \\
\text { de evidencia moderado. }\end{array}$ \\
\hline $\begin{array}{l}\text { 12. Después de la falla a la terapia TCB, una terapia } \\
\text { triple o cuádruple con una fluoroquinolona puede ser } \\
\text { recomendada. En caso de alta resistencia a las } \\
\text { quinolonas, la combinación de bismuto con otros } \\
\text { antibióticos o rifabutina puede ser una opción. }\end{array}$ & $\begin{array}{l}\text { Recomendación débil, nivel de } \\
\text { evidencia muy bajo. }\end{array}$ \\
\hline $\begin{array}{l}\text { 13. Después de la falla a la TTE, una terapia cuádruple } \\
\text { que contenga Bismuto o una terapia triple o cuádruple } \\
\text { con fluoroquinolonas puede ser recomendada como una } \\
\text { segunda línea de tratamiento. }\end{array}$ & $\begin{array}{l}\text { Recomendación débil, nivel de } \\
\text { evidencia bajo. }\end{array}$ \\
\hline $\begin{array}{l}\text { 14. Después de la falla a la terapia cuádruple sin } \\
\text { Bismuto, tanto la terapia TCB como la terapia triple o } \\
\text { cuádruple con quinolonas puede ser recomendada. }\end{array}$ & $\begin{array}{l}\text { Recomendación débil, nivel de } \\
\text { evidencia muy bajo. }\end{array}$ \\
\hline $\begin{array}{l}\text { 15. Después de la falla al tratamiento con una terapia de } \\
\text { segunda linea, un cultivo con examen de susceptibilidad } \\
\text { o determinación del genotipo de resistencia es } \\
\text { recomendado para guiar el tratamiento. }\end{array}$ & $\begin{array}{l}\text { Recomendación débil, nivel de } \\
\text { evidencia muy bajo. }\end{array}$ \\
\hline $\begin{array}{l}\text { 16. Después de la falla a los tratamientos de primera línea } \\
\text { (basados en claritromicina) y a tratamientos de segunda } \\
\text { linea (terapia TCB) se recomienda el uso de regimenes } \\
\text { con fluoroquinolonas. En los lugares con alta resistencia } \\
\text { a fluoroquinolonas, se recomienda una combinación de } \\
\text { Bismuto con diferentes antibióticos o la terapia de rescate } \\
\text { con rifabutina puede ser considerada. }\end{array}$ & $\begin{array}{l}\text { Recomendación débil, nivel de } \\
\text { evidencia muy bajo. }\end{array}$ \\
\hline $\begin{array}{l}\text { 17. Después de la falla al tratamiento de primera línea } \\
\text { (triple o cuádruple sin bismuto) y a terapias de segunda } \\
\text { línea (terapia con fluoroquinolonas), se recomienda el } \\
\text { tratamiento con terapia TCB. }\end{array}$ & $\begin{array}{l}\text { Recomendación débil, nivel de } \\
\text { evidencia muy bajo. }\end{array}$ \\
\hline $\begin{array}{l}\text { 18. Después de la falla a la primera línea de tratamiento } \\
\text { con terapia TCB y terapia de segunda línea (terapia con } \\
\text { fluoroquinolona), se recomienda el uso de terapia triple o } \\
\text { cuádruple con claritromicina. Una combinación de } \\
\text { Bismuto con otros antibióticos puede ser una alternativa. }\end{array}$ & $\begin{array}{l}\text { Recomendación débil, nivel de } \\
\text { evidencia muy bajo. }\end{array}$ \\
\hline $\begin{array}{l}\text { 19. En pacientes con alergia a la penicilina, en áreas con } \\
\text { baja resistencia a la claritromicina una combinación de } \\
\text { IBP, claritromicina y metronidazol puede ser utilizada. Y } \\
\text { en áreas con alta resistencia a la claritromicina, la terapia } \\
\text { TCB debe ser preferida. }\end{array}$ & $\begin{array}{l}\text { Recomendación débil, nivel de } \\
\text { evidencia muy bajo. }\end{array}$ \\
\hline $\begin{array}{l}\text { 20. Un régimen de rescate que contenga una } \\
\text { fluoroquinolona puede ser una segunda línea de manejo } \\
\text { empirica en la presencia de alergia a la penicilina. }\end{array}$ & $\begin{array}{l}\text { Recomendación débil, nivel de } \\
\text { evidencia muy bajo. }\end{array}$ \\
\hline
\end{tabular}

Tabla 5

Recomendaciones de la Guía Colombiana de Práctica Clínica (25)

\begin{tabular}{|c|c|}
\hline Recomendación & $\begin{array}{c}\text { Nivel de } \\
\text { evidencia }\end{array}$ \\
\hline $\begin{array}{l}\text { Se recomienda el tratamiento de primera linea con base en las pruebas de } \\
\text { susceptibilidad. }\end{array}$ & \begin{tabular}{|ll} 
Bajo \\
\end{tabular} \\
\hline $\begin{array}{l}\text { Se sugiere que el tratamiento de segunda línea no se haga con base en los } \\
\text { resultados de pruebas de susceptibilidad. }\end{array}$ & Muy bajo \\
\hline $\begin{array}{l}\text { Se recomienda que los esquemas de erradicación del } H \text {. pylori tengan una } \\
\text { duración de } 14 \text { días. }\end{array}$ & Bajo \\
\hline $\begin{array}{l}\text { Se recomienda la terapia secuencial o la terapia triple estándar durante } 14 \\
\text { días como tratamiento de primera línea. }\end{array}$ & Muy bajo \\
\hline $\begin{array}{l}\text { Se sugiere cualquier inhibidor de bomba de protones como parte de la } \\
\text { terapia de erradicación. }\end{array}$ & Bajo \\
\hline $\begin{array}{l}\text { Como segunda línea de tratamiento, se recomienda utilizar por lo menos } 2 \\
\text { antibióticos distintos a los utilizados en la primera linea. }\end{array}$ & Muy bajo \\
\hline $\begin{array}{l}\text { Se sugiere terapia triple con moxifloxacina, levofloxacina o cuádruple con } \\
\text { bismuto si estos no fueron utilizados en la primera linea de tratamiento. }\end{array}$ & Muy bajo \\
\hline $\begin{array}{l}\text { Se sugiere la utilización de probióticos o rebamipida como coadyuvantes } \\
\text { para la erradicación del } H \text {. pylori. }\end{array}$ & Moderado \\
\hline $\begin{array}{l}\text { Se sugiere que los pacientes reciban tratamiento periodontal, además de la } \\
\text { terapia de erradicación del H. pylori para disminuir la persistencia de este } \\
\text { microorganismo. }\end{array}$ & Bajo \\
\hline $\begin{array}{l}\text { Se recomienda que las terapias de erradicación en zonas de alta resistencia a } \\
\text { los antibióticos básicos (claritromicina, metronidazol) sean la terapia } \\
\text { cuádruple con bismuto o terapias concomitantes. }\end{array}$ & Bajo \\
\hline $\begin{array}{l}\text { Se sugiere utilizar } 1 \text { dosis de claritromicina de } 500 \mathrm{mg} \text { al día, en vez de la } \\
\text { misma dosis } 2 \text { veces al día. }\end{array}$ & Bajo \\
\hline $\begin{array}{l}\text { Se sugiere tratamiento de erradicación del } H \text {. pylori para disminuir la } \\
\text { incidencia global del cáncer gástrico en todos los pacientes en quienes se } \\
\text { identifique esta infección. }\end{array}$ & Moderado \\
\hline $\begin{array}{l}\text { Se recomienda la erradicación del } H \text {. pylori en pacientes con enfermedad } \\
\text { por reflujo gastroesofágico porque no modifica los síntomas de esta última } \\
\text { entidad. }\end{array}$ & \begin{tabular}{|l|} 
Muy Bajo \\
\end{tabular} \\
\hline Se recomienda la erradicación del $H$. pylori en pacientes con dispepsia. & Moderado \\
\hline $\begin{array}{l}\text { Se recomienda investigar y erradicar el } H \text {. pylori en todos los pacientes con } \\
\text { antecedente de úlcera péptica complicada o no, con sangrado. }\end{array}$ & \begin{tabular}{|l} 
Bajo \\
\end{tabular} \\
\hline $\begin{array}{l}\text { Se recomienda la erradicación del } H \text {. pylori en pacientes que van a iniciar el } \\
\text { consumo crónico de AINES o aspirina. }\end{array}$ & Bajo \\
\hline $\begin{array}{l}\text { Se recomienda la erradicación del } H \text {. pylori en pacientes con antecedente de } \\
\text { linfoma MALT de bajo grado, con compromiso confinado a la mucosa y la } \\
\text { submucosa, y en quienes presenten la mutación de gen API2-MALT1. }\end{array}$ & Bajo \\
\hline $\begin{array}{l}\text { Se recomienda la erradicación del } H \text {. pylori en pacientes con anemia } \\
\text { ferropénica a quienes se les hayan descartado las otras causas de esta } \\
\text { anemia. }\end{array}$ & Bajo \\
\hline
\end{tabular}

\section{Desenlaces no mencionados en las guías}

Chen y cols. (44) encontraron que las tasas de erradicación de los pacientes con terapia dirigida por tasas de resistencia locales fueron mayores que en aquellos con terapia empírica (RR: 1,16; IC95\%: 1,10-1,22). Por otro lado, las terapias guiadas por susceptibilidad obtuvieron mayor erradicación que las terapias empíricas; las terapias basadas en pruebas moleculares y cultivos fueron mejores que la terapia empírica (molecular, RR: 1,3; IC95\%: 1,11-1,35; cultivo, RR: 1,13; IC95\%: 1,06-1,20).

Según el metanálisis realizado por Bang y cols. (45), la erradicación del H. pylori posterior a la resección endoscópica de cáncer gástrico temprano redujo la probabilidad de desarrollar cáncer gástrico metacrónico. 


\section{Discusión}

Han pasado 20 años desde el descubrimiento del $\mathrm{H}$. pylori como agente causal del cáncer gástrico, con importantes avances respecto a su diagnóstico y manejo; se han identificado dos genotipos importantes (i. e., VacA y CagA), siendo el genotipo CagA el más patógeno (49).

La eficacia de las opciones consideradas de primera línea de manejo ha disminuido progresivamente, al punto que los regímenes utilizados en la práctica clínica se asumen como subterapéuticos (28). La preocupación por lograr índices de erradicación superiores al $90 \%$ ha llevado a contemplar diferentes regímenes antibióticos y terapias coadyuvantes (i. e., probióticos o tratamientos periodontales), sin que esto haya demostrado una mayor efectividad, a pesar de una diferencia en costos y en disminución de efectos adversos. En consecuencia, la investigación en este campo ha crecido de manera exponencial, procurando dar respuesta al reto de controlar la infección por este microorganismo considerado como un carcinógeno tipo 1 (6).

El diagnóstico de la infección por $H$. pylori debe ser una prioridad en salud pública. Así, aun cuando se realiza por medio de endoscopia de vías digestivas altas con biopsia para análisis histológico y pruebas de ureasa (26), en poblaciones de alto riesgo es posible la toma de cultivos con antibiograma para orientar específicamente el tratamiento, teniendo en cuenta la evidencia de mayor probabilidad de erradicación con un tratamiento individualizado. El tratamiento debe ser el más eficaz posible para evitar la inducción de resistencia bacteriana. Estas pruebas también deben llevarse a cabo en pacientes con resistencia a terapia empírica, en quienes se deben incluir por lo menos dos antibióticos diferentes a los usados en la primera línea, para garantizar el éxito del siguiente esquema de manejo (25).

Posterior al tratamiento, se recomienda evaluar la efectividad utilizando el test de aliento con urea marcada o los antígenos fecales, y según el resultado tomar medidas adicionales (25). Si bien un resultado positivo puede indicar recrudescencia de la infección, definida como la reaparición de la infección inicial que estuvo temporalmente indetectable como un falso positivo de erradicación, y por tanto indicar resistencia antimicrobiana, también existe la posibilidad de que se trate de una reinfección (50). Un metanálisis reciente calculó una tasa global anual de reinfección cercana al 5\%, que incluso puede incrementarse de manera inversamente proporcional según el Índice de Desarrollo Humano, hasta alcanzar cifras cercanas al 11\% (50). Esto evidencia la importancia de considerar la ocurrencia de reinfección en los países de medianos y bajos ingresos, como Colombia.

La tasa global de resistencia del $\mathrm{H}$. pylori se ha reportado en un 17,2\%, (IC95\%: 16,5-17,9) para claritromicina y en un 26,7\% (IC95\%: 25,2-28,1) para metronidazol (11,2\%; IC95\%: 9,6-12,7). La resistencia a múltiples antibióticos es cercana al 10\% (51). Los consensos de expertos proponen utilizar un punto de corte del $15 \%$ para la resistencia a claritromicina y del $40 \%$ para metronidazol, como límite superior para el uso de terapias que contengan estos medicamentos como base del tratamiento (47).

En la revisión sistemática realizada por Camargo y cols. (52) se calculó la resistencia antibiótica en Latinoamérica. Para Colombia se analizaron 11 estudios que calcularon la resistencia a claritromicina en un $18 \%$; mientras que para metronidazol dos estudios mostraron una resistencia del 83\%. Estos incluyeron pocos pacientes, provenientes en su mayoría de centros hospitalarios, lo que pudo asociarse con sesgos importantes. No obstante, si se tomaran de forma estricta los valores reportados y siguiendo los consensos nacionales e internacionales de manejo, la TTE no sería de primera línea en nuestro país. Varias consideraciones deben tenerse en cuenta para plantear alternativas.

En primer lugar, la terapia SEC surge como una alternativa a la resistencia evidenciada; estudios demuestran que esta terapia, administrada durante 14 días, es superior a la TTE por este mismo tiempo (39). Considerando esta evidencia, la guía colombiana la propone como alternativa de primera línea (25). Sin embargo, 
los resultados de algunos estudios indican menor efectividad cuando la terapia se administra a lo largo de 10 días $(28,53)$; adicionalmente, después del 2008 se ha reportado una disminución de la eficacia del tratamiento SEC durante 10 días en comparación con el tratamiento con TTE por el mismo tiempo (28). Dados tales motivos, el Consenso de Toronto recomienda no usarlo (46). Adicionalmente, el Consenso de Maastritch V prioriza las terapias CON y TTE como primera línea de manejo (47).

La implementación de terapias coadyuvantes para aumentar la efectividad, como la terapia periodontal, basada en la disminución de la colonización oral como factor fundamental para la disminución de recurrencias (54), la profilaxis oral y la eliminación de placas bacterianas han mostrado ser estrategias benéficas, particularmente en pacientes con colonización en mucosa oral. Para la guía colombiana, estas son las intervenciones recomendadas antes del tratamiento, durante este o después (25). Los probióticos tienen un efecto positivo en la erradicación del $\mathrm{H}$. pylori (33), debido a que inducen mecanismos antinflamatorios y antioxidativos que regulan la microbiota intestinal. El beneficio de administrar probióticos no solo se relaciona con mayor tasa de erradicación, sino con la reducción de efectos adversos (43). El consenso de Maastricht $\mathrm{V}$ señala que el uso de $\mathrm{S}$. boulardii podría implementarse, a pesar de una evidencia débil (47). En contraste, el Consenso de Toronto 2016 no lo recomienda (46). La guía colombiana considera los probióticos como coadyuvantes que pueden favorecer el tratamiento antibiótico (25).

Lo anterior trae a colación la discusión de si actualmente en Colombia estamos tratando la infección de la manera más adecuada. Según la evidencia más reciente, consideramos que, tomando como base las tasas de resistencia reportadas, la terapia SEC durante 14 días debe ser de primera línea de tratamiento en nuestro medio, tal como lo propone la guía colombiana (25). Hacemos hincapié en la importancia de motivar a que el paciente se adhiera al tratamiento completo, ya que el cambio de medicamentos a los 7 días puede traer problemas en este sentido; también se recomienda la administración de probióticos y la realización de terapia periodontal sistemática, esta última teniendo en cuenta su cobertura universal en nuestro sistema de aseguramiento. Estas medidas podrían aumentar la eficacia y, eventualmente, disminuir la resistencia antibiótica. Es prioritaria la realización de estudios de costo-efectividad que permitan la elección del esquema ideal en nuestro medio por parte de los tomadores de decisiones.

Aunque nuestra consideración es contraria a lo propuesto por los consensos canadiense y europeo, siempre deben tenerse en cuenta las diferencias regionales y, por tanto, en Colombia la terapia SEC durante 14 días es una alternativa válida, tal como lo plantea la guía colombiana (25).

Por otro lado, una opción en áreas con baja resistencia a la TTE es el uso de claritromicina a mitad de dosis (29), consideración también recomendada por la guía colombiana $(500$ mg de claritromicina al día en vez de $1 \mathrm{~g} /$ día) (25). Resaltamos que las terapias con fluoroquinolonas, sin bien han mostrado ser efectivas (24), en países endémicos de tuberculosis, como el caso de Colombia, deben usarse con cautela, dado que estos antimicrobianos hacen parte del tratamiento de segunda línea para esta patología, la cual enfrenta un incremento en sus tasas de resistencia (55).

Finalmente, vale la pena resaltar la importancia de la identificación y tratamiento temprano, es decir, antes del desarrollo de lesiones premalignas. Ford y cols. (26) no encontraron evidencia de disminución en el riesgo de desarrollar cáncer gástrico posterior al tratamiento, cuando los pacientes tenían lesiones premalignas (RR: 0,86; IC95\% 0,47-1,59). Este fenómeno es conocido como la "teoría del no retorno" y debe ser extensamente conocido por los profesionales de la salud.

\section{Conclusiones}

La efectividad de las opciones terapéuticas de erradicación del H. pylori ha disminuido, debido 
al incremento en la resistencia antimicrobiana, por lo que actualmente se sugieren terapias guiadas por patrones de resistencia. Esta estrategia es limitada en Colombia, dada la escasez de estudios de resistencia locales. El uso adecuado de medicamentos podría disminuir los costos para el sistema de salud, disminuir la resistencia antimicrobiana y ayudar a erradicar efectivamente las cepas patógenas. La SEC durante 14 días surge como una alternativa razonable en contextos con alta resistencia a claritromicina y metronidazol; pero se requieren más estudios epidemiológicos y de costoefectividad para sustentar estas recomendaciones de forma sistemática.

\section{Referencias}

1. Blaser MJ, Atherton JC. Helicobacter pylori persistence: Biology and disease. J Clin Invest. 2004;113(3):321-33.

2. Correa P. A human model of gastric carcinogenesis. Cancer Res. 1988;48(13):3554-60.

3. Correa P, Piazuelo MB. Helicobacter pylori infection and gastric adenocarcinoma. US Gastroenterol Hepatol Rev. 2011;7(1):59-64.

4. Sugano K, Tack J, Kuipers EJ, Graham DY, El-Omar EM, Miura S, et al. Kyoto global consensus report on Helicobacter pylori gastritis. Gut. septiembre de 2015;64(9):1353-67.

5. Forman D, Newell DG, Fullerton F, Yarnell JW, Stacey AR, Wald N, et al. Association between infection with Helicobacter pylori and risk of gastric cancer: evidence from a prospective investigation. BMJ. 1991;302 (6788):1302-5.

6. IARC Working Group on the Evaluation of Carcinogenic Risks to Humans. Schistosomes, liver flukes and Helicobacter pylori. IARC Monogr Eval Carcinog risks to humans. 1994;61:1-241.
7. Hooi JKY, Lai WY, Ng WK, Suen MMY, Underwood FE, Tanyingoh D, et al. Global Prevalence of Helicobacter pylori infection: Systematic review and meta-analysis. Gastroenterology. 2017 Ago;153(2):420-9.

8. Otero WR. Helicobacter pylori en agua potable: $i E s$ la ruta de la infección? Acta Med Colomb. 2017;42(2):87-9.

9. Klein PD, Graham DY, Gaillour A, Opekun AR, Smith EO. Water source as risk factor for Helicobacter pylori infection in Peruvian children. Gastrointestinal Physiology Working Group. Lancet. 1991 jun 22;337(8756):1503-6.

10. Aziz RK, Khalifa MM, Sharaf RR. Contaminated water as a source of Helicobacter pylori infection: A review. J Adv Res. 2015 jul;6(4):539-47.

11. Bellack NR, Koehoorn MW, MacNab YC, Morshed MG. A conceptual model of water's role as a reservoir in Helicobacter pylori transmission: A review of the evidence. Epidemiol Infect. 2006 jun;134(3):439-49.

12. Goh K-L, Chan W-K, Shiota S, Yamaoka Y. Epidemiology of Helicobacter pylori infection and public health implications. Helicobacter. 2011 sep;16 Suppl 1:1-9.

13. Khalifa MM, Sharaf RR, Aziz RK. Helicobacter pylori: A poor man's gut pathogen? Gut Pathog. 2010 marz 21;2(1):2.

14. Cuervo CM, Gaviria AM. Detección de Helicobacter pylori en muestras de agua y biopelícula de los grifos de las instituciones educativas oficiales en la ciudad de Medellín. Acta Med Colomb. 2017;42(2):121-8.

15. Zeng M, Mao XH, Li JX, Tong W De, Wang B, Zhang YJ, et al. Efficacy, safety, and immunogenicity 
of an oral recombinant Helicobacter pylori vaccine in children in China: a randomised, double-blind, placebocontrolled, phase 3 trial. Lancet. 2015;386(10002):1457-64.

16. Fuccio L, Zagari RM, Eusebi LH, Laterza L, Cennamo V, Ceroni L, et al. Meta-analysis: can Helicobacter pylori eradication treatment reduce the risk for gastric cancer? Ann Intern Med. 2009;151(2):121-8.

17. Malfertheiner P, Megraud F, O'Morain CA, Atherton J, Axon ATR, Bazzoli F, et al. Management of Helicobacter pylori infection--the Maastricht IV/ Florence Consensus Report. Gut. 2012;61(5):646-64.

18. Gisbert JP, Calvet X. Review article: the effectiveness of standard triple therapy for Helicobacter pylori has not changed over the last decade, but it is not good enough. Aliment Pharmacol Ther. 2011;34(11-12):1255-68.

19. Qasim A, Sebastian S, Thornton O, Dobson M, McLoughlin R, Buckley M, et al. Rifabutin- and furazolidone-based Helicobacter pylori eradication therapies after failure of standard first- and second-line eradication attempts in dyspepsia patients. Aliment Pharmacol Ther. 2005;21(1):91-6.

20. Gatta L, Zullo A, Perna F, Ricci C, De Francesco V, Tampieri A, et al. A 10-day levofloxacin-based triple therapy in patients who have failed two eradication courses. Aliment Pharmacol Ther. 2005;22(1):45-9.

21. Gisbert JP, Gonzalez L, Calvet X. Systematic review and meta-analysis: proton pump inhibitor vs. ranitidine bismuth citrate plus two antibiotics in Helicobacter pylori eradication. Helicobacter. 2005;10(3):157-71.

22. Fischbach LA, van Zanten S, Dickason J. Meta-analysis: the efficacy, adverse events, and adherence related to first-line anti-Helicobacter pylori quadruple therapies. Aliment Pharmacol Ther. 2004;20(10):1071-82.

23. He L, Deng T, Luo H. Metaanalysis of sequential, concomitant and hybrid therapy for Helicobacter pylori eradication. Intern Med. 2015;54(7):703-10.

24. Kale-Pradhan PB, Mihaescu A, Wilhelm SM. Fluoroquinolone sequential therapy for Helicobacter pylori: A meta-analysis. Pharmacotherapy. 2015;35(8):719-30.

25. Otero R W, Trespalacios R AA, Otero P L, Vallejo O MT, Torres Amaya M, Pardo R, et al. Clinical practice guideline for the diagnosis and management of adult patients with Helicobacter pylori infection. Rev Colomb Gastroenterol. 2015;30 (Suppl. 1):17-33.

26. Ford AC, Forman D, Hunt R, Yuan Y, Moayyedi P. Helicobacter pylori eradication for the prevention of gastric neoplasia. Cochrane Database Syst Rev. 2015;(7):CD005583.

27. Ford AC, Gurusamy KS, Delaney B, Forman D, Moayyedi P. Eradication therapy for peptic ulcer disease in Helicobacter pylori-positive people. Cochrane Database Syst Rev. 2016;4:CD003840.

28. Nyssen OP, McNicholl AG, Megraud F, Savarino V, Oderda G, Fallone CA, et al. Sequential versus standard triple first-line therapy for Helicobacter pylori eradication. Cochrane Database Syst Rev. 2016; (6):CD009034 .

29. Harb AH, Chalhoub JM, Abou Mrad R, Sharara AI. Systematic review and meta-analysis: full- vs. halfdose anti-microbials in clarithromycinbased regimens for Helicobacter pylori 
eradication. Aliment Pharmacol Ther. 2015;42(2):131-41.

30. Hsu PI, Lin PC, Graham DY. Hybrid therapy for Helicobacter pylori infection: A systemic review and meta-analysis. World J Gastroenterol. 2015;21(45):12954-62.

31. Kim JS, Park SM, Kim B-W. Sequential or concomitant therapy for eradication of Helicobacter pylori infection: A systematic review and meta-analysis. J Gastroenterol Hepatol. 2015;30(9):1338-45.

32. Lee SW, Kim HJ, Kim JG. Treatment of Helicobacter pylori Infection in Korea: A systematic review and meta-analysis. J Korean Med Sci. 2015;30(8):1001-9.

33. Li BZ, Threapleton DE, Wang JY, Xu JM, Yuan JQ, Zhang C, et al. Comparative effectiveness and tolerance of treatments for Helicobacter pylori: Systematic review and network meta-analysis. BMJ. 2015;351:h4052.

34. Losurdo G, Leandro G, Principi M, Giorgio F, Montenegro L, Sorrentino C, et al. Sequential vs. prolonged 14-day triple therapy for Helicobacter pylori eradication: the meta-analysis may be influenced by "geographical weighting". Int J Clin Pract. 2015;69(10):1112-20.

35. López-Góngora S, Puig I, Calvet X, Villoria A, Baylina M, Muñoz N, et al. Systematic review and meta-analysis: susceptibilityguided versus empirical antibiotic treatment for Helicobacter pylori infection. J Antimicrob Chemother. 2015 sep;70(9):2447-55.

36. Lv ZF, Wang FC, Zheng HL, Wang B, Xie Y, Zhou XJ, et al. Metaanalysis: Is combination of tetracycline and amoxicillin suitable for Helicobacter pylori infection? World J Gastroenterol. 2015;21(8):2522-33.
37. Teng M, Khoo AL, Zhao YJ, Lin L, Lim BP, Wu TS, et al. Meta-analysis of the effectiveness of esomeprazole in gastroesophageal reflux disease and Helicobacter pylori infection. J Clin Pharm Ther. 2015;40(4):368-75.

38. Wang B, Wang YH, Lv ZF, Xiong HF, Wang $\mathrm{H}$, Yang $\mathrm{Y}$, et al. Efficacy and safety of hybrid therapy for Helicobacter pylori infection: A systematic review and meta-analysis. Helicobacter. 2015;20(2):79-88.

39. Liou JM, Chen CC, Lee YC, Chang CY, Wu JY, Bair MJ, et al. Systematic review with meta-analysis: 10 - or 14 day sequential therapy vs. 14-day triple therapy in the first line treatment of Helicobacter pylori infection. Aliment Pharmacol Ther. 2016;43(4):470-81.

40. Niv Y. Doxycycline in eradication therapy of Helicobacter pylori: A systematic review and meta-analysis. Digestion. 2016;93(2):167-73.

41. Ren Q, Yan X, Zhou Y, Li WX. Periodontal therapy as adjunctive treatment for gastric Helicobacter pylori infection. Cochrane Database Syst Rev. 2016;2:CD009477.

42. Szajewska H, Horvath A, Kołodziej M. Systematic review with meta-analysis: Saccharomyces boulardii supplementation and eradication of Helicobacter pylori infection. Aliment Pharmacol Ther. 2015;41(12):1237-45.

43. Zhang MM, Qian W, Qin YY, He J, Zhou YH. Probiotics in Helicobacter pylori eradication therapy: A systematic review and meta-analysis. World J Gastroenterol. 2015;21(14):4345-57.

44. Chen H, Dang Y, Zhou X, Liu B, Liu S, Zhang G. Tailored therapy versus empiric chosen treatment for Helicobacter pylori eradication: A meta-analysis. Medicine (Baltimore). 2016;95(7):e2750. 
45. Bang CS, Baik GH, Shin IS, Kim JB, Suk KT, Yoon JH, et al. Helicobacter pylori eradication for prevention of metachronous recurrence after endoscopic resection of early gastric cancer. J Korean Med Sci. 2015;30(6):749-56.

46. Fallone CA, Chiba N, van Zanten SV, Fischbach L, Gisbert JP, Hunt $\mathrm{RH}$, et al. The Toronto Consensus for the Treatment of Helicobacter pylori infection in adults. Gastroenterology. 2016;151(1):51-69.e14.

47. Malfertheiner P, Megraud F, O'Morain CA, Gisbert JP, Kuipers EJ, Axon AT, et al. Management of Helicobacter pylori infection-the Maastricht V/Florence Consensus Report. Gut. 2017;66(1):6-30.

48. Fashner J, Gitu AC. Diagnosis and treatment of peptic ulcer disease and H. pylori infection. Am Fam Physician. 2015;91(4):236-42.

49. Mobley HL. Defining Helicobacter pylori as a pathogen: Strain heterogeneity and virulence. Am J Med. 1996;100(5A):2S-9S; discussion 9S-11S.

50. Hu Y, Wan J-H, Li X-Y, Zhu Y, Graham DY, Lu N-H. Systematic review with meta-analysis: The global recurrence rate of Helicobacter pylori. Aliment Pharmacol Ther. noviembre de 2017;46(9):773-9.

51. De Francesco V, Giorgio F, Hassan C, Manes G, Vannella L, Panella C, et al. Worldwide $H$. pylori antibiotic resistance: A systematic review. J Gastrointest Liver Dis. 2010;19(4):409-14.

52. Camargo MC, García A, Riquelme A, Otero W, Camargo CA, HernándezGarcía $\mathrm{T}$, et al. The problem of Helicobacter pylori resistance to antibiotics: A systematic review in
Latin America. Am J Gastroenterol. 2014;109(4):485-95.

53. Gatta L, Vakil N, Vaira D, Scarpignato C. Global eradication rates for Helicobacter pylori infection: Systematic review and metaanalysis of sequential therapy. BMJ. 2013;347:f4587.

54. Zou QH, Li RQ. Helicobacter pylori in the oral cavity and gastric mucosa: A meta-analysis. J oral Pathol Med. 2011;40(4):317-24.

55. Pai M, Behr MA, Dowdy D, Dheda K, Divangahi M, Boehme CC, et al. Tuberculosis. Nat Rev Dis Prim. 2016;2:16076. 